\title{
Ein Masterplan für die kreative Stadt
}

\author{
Für eine Wiedergewinnung des Demokratischen
}

Konrad Hummel

Viele gesellschaftliche, wirtschaftliche und kulturelle Probleme der Gegenwart zeigen sich in den Großstädten wie unter einem Brennglas. Mit dem Leitbild der »kreativen Stadt " kann das Gemeinwesen wieder zum » Geburtsort der Freibeit " (Mitscherlich) werden, in dem Menschen in Verbundenheit kreativ sich engagieren.

Das Bild einer »kreativen Stadt « steht für eine soziale Stadtentwicklung, die den Herausforderungen einer öffentlich verarmten, dem Globalisierungswettbewerb ohnmächtig ausgelieferten, der sozial und nach Ethnien und Generationen zersplitterten Stadt erfolgreich zu widerstehen scheint.

Ist die kreative Stadt ein Hoffnungsbild, wie eine moderne Wissensgesellschaft in der Stadt, die ihre Sachzwänge und Blockaden überwindet? Gelingt es in der kreativen Stadt das Soziale als das Verbindende einer Stadtgesellschaft neu zu entdecken trotz der Krise und dem Umbau des Sozialstaates?

Tatsächlich unternehmen wir den Versuch, die Neuaufstellung des (überwiegend) alten städtischen Orchesters mit zusätzlichen Akteuren und neuen Weisen und Melodien als im Ergebnis kreative, gelingende Wiederentdeckung des sozialen Klangs der europäischen Stadt zu bezeichnen.

In der kreativen Stadt werden Institutionen aktiviert, geöffnet, neu einander zugeordnet, ergänzt - wie das Arrangement der Instrumente im Orchester. Wir setzen nicht daran an, uns die Menschen anders zu wünschen oder völlig neue Dienstleistungen auszubauen sondern beispielsweise Fachkräfte und Freiwillige, Betroffene und Nachbarn, Organisierte

Dr. Konrad Hummel ist Sozial- und Jugendreferent der Stadt Augsburg. Er ist Mitglied im Beirat der Blätter der Wohlfahrtspflege.

E-Mail sozialreferat@augsburg.de und spontane Formen der Teilhabe sinnvoll und neu zu mischen.

Moderne Formen der Kinderbetreuung zeigen dies beispielhaft. Eigenes und nachbarschaftliches Handeln, kombiniert von fachlichen und mobilen Diensten, wohnungswirtschaftlichen und gesundheitsorientierten Leistungen ergeben einen Wohlfahrtsmix, der bunt aber nicht beliebig ist.

Wie der Klang einer offenen, toleranten, solidarischen Stadtgesellschaft sich anhören sollte, haben viele beschrieben. Wenige haben die Methoden und Leitprinzipien beschrieben, wie dies bewerkstelligt werden kann. Solange überwiegen die Wunschbilder (und spiegelbildlich ihre Kritiker) und vor allem Appelle nach Tugend und Moral.

Ein besseres Miteinander von jung und alt, von deutsch und ausländisch, von arm und reich, von Mann und Frau, gesund und behindert wird beschworen, viele Einzelbeispiele als »Best Practice« dargestellt. Das Bedürfnis in der Gesellschaft nach solchen positiven Verhaltensbeispielen ist groß, weil der Sozialstaat seit jeher mehr ist als ein Tauschgeschäft von Gebern und Empfängern. Der Sozialstaat trägt immer ein Stück »Utopie« (1) vom gerechten Lastenausgleich in sich und besonders in Zeiten des Wandels und Umbruchs als Symbol für die Temperatur einer Gesellschaft.

\section{Demokratie und ihre Institutionen}

Transmissionsriemen zwischen Gesellschaftsentwurf und Alltag der Menschen sind in einer modernen Stadtgesellschaft die Institutionen. Ohne sie funktionieren weder Alltag noch erwartbare und nachhaltige Verhaltensweisen und Änderungen. Walzer formuliert es für die Toleranz in modernen Gesellschaften so, dass sie funktionieren muss, gleich wie ihre Motive, ihre Ausprägung, ihr Enthusiasmus ist - entscheidend ist, dass sie mittels guter Institutionen zum Tragen kommt. (2)
Mit Institutionen sind in einem weiteren Sinne nicht einzelne Schulen oder Kindergärten gemeint, sondern die Regelwerke der Wirtschaft, des Sozialstaates, der Stadt, der Nachbarschaft und gesellschaftlicher Vereine und Vereinigungen. Sie folgen inneren Ordnungen. Und sie bewirken, dass bestimmte Grundwerte umgesetzt und verwirklicht werden.

- Wirtschaftsorientierte Einrichtungen stehen für Dienstleistungsorientierung und sollen Eigenverantwortung der Kunden stärken.

- Staatliche Institutionen sichern eine formale Art von Solidarität, in der niemand rausfällt.

- Städtische Institutionen können und sollen Vielfalt sichern, weil es hier jeweils - etwa im Sozialraum - um alle dort Lebenden geht, gleich ob sie formale Rechte haben, über Kaufkraft verfügen oder eine Familie haben.

- Städtische Nachbarschaft steht für Unmittelbarkeit, Selbsthilfe und soziale Kontrolle. Wo diese nicht bestehen kann, treten die unmittelbaren Versorgungsinstitutionen ein wie Alten- oder Kinderheime etc.

- Das Vereinswesen schließlich steht für den Gemeinsinn, Gemeinschaft und die Interessenvertretung der Gruppen.

Dieses unsichtbar aufeinander abgestimmte Gefüge korrespondiert mit einer Gesellschaft, in der es vorherrschende Bilder von Familie, Lebenslauf, Erwerbsarbeit und Integration von Minderheiten gibt. In einer sich still und dramatisch ändernden Gesellschaft der Kleinstfamilien und des Älterwerdens, der veränderten und mobilen Erwerbsarbeit, Migration, Lebensstile, funktioniert das Zusammenspiel von Institutionen und gesellschaftlich vermittelten Werten nicht mehr.

Im gesellschaftlichen Wandel vor drei Jahrzehnten ging es um die Öffnung verkrusteter Institutionen, um dem Aufbruch der Produktivkräfte und den neuen Lebensstilen Raum zu geben. (3) Wir schrieben »damals « von der Öffnung der 
Heime, der Deinstitutionalisierung. (4) Letztlich ist dies so umfassend geschehen, dass die Kehrseite freigesetzter »Wirtschaftskräfte « in den Städten als unmittelbarer »Wärmeverlust « spürbar geworden ist. Marktwirtschaft instrumentalisiert Gefühle, nimmt aber die solidarischen Gefühle von Gerechtigkeit und Rücksicht nicht mit auf.

Der Sozialstaat hat sich der Veränderung der Gesellschaft der letzten 20 Jahre erwehrt durch Rückschnitte auf das »unmittelbar Notwendige ", auf schärfere Effizienzkontrollen mit der Folge, dass er völlig im Teufelskreis gefangen ist für immer mehr Geld, immer weniger und »einzelfallteure« Klienten zu bedienen. So wird der Sozialstaat noch bestenfalls als Versicherungsagentur aber nicht mehr als Solidaritätsstifter verstanden. Der Druck auf die europäische Stadt hat zugenommen, selbst Ausfallbürge für den sozialen Staat zu sein. Die Arbeitsmarktreform Hartz IV zeigt, dass die Kommunen inzwischen auch Arbeitsmarktpolitik stärker selbst machen.

\section{Zerfällt die europäische Stadt?}

Der Stadt ihrerseits wurden in den letzten Jahren immer mehr Instrumente aus der Hand genommen, Vielfalt erlebbar und organisierbar zu machen. Wahlfreiheit ermöglicht vielen Familien sich »freizukaufen « von Daseinseinrichtungen im Stadtteil.

- Privatschulen, ethnische Sonderkinderund Senioreneinrichtungen drohen Sozialräume zu sprengen.

- Wettbewerbsvorschriften drängen Städte, ihre Verkehrs-, Abwasser-, Wohnungs- und andere Einrichtungen zu verkaufen, sodass die integrierende Stadt immer weniger erlebbar wird und sich umso mehr in Kulturevents inszeniert. Nachbarschaften werden anonymer und gleichzeitig ethnisch homogener.

- Der türkisch oder russische Jugendliche der zweiten oder dritten Zuwanderungsgeneration bewegt sich im eigenen Supermarkt und Disco und ist nicht mehr angehalten, deutsch zu sprechen. Die doppelt so große schulische Versagensquote dieser Generation schlägt still und schmerzhaft bis in den Stadtteil zurück, weil dessen öffentlicher Bolz- und Kirchplatz zum ersten Erlebnisort des arbeitslosen Jugendlichen wird.
- Die von uns geöffneten Institutionen stehen unter dem Knebel der Belegplatzfinanzierung, Spezialisierung und einem gezielten Bildungs- und Rehaauftrag, in dem beispielsweise freiwillige Helfer eher stören. Die Logik der Rationalisierung verdrängt Bürgerengagement. Diese Gefahr ist ungleich größer, als durch Engagement-Fachstellen einsparen zu wollen.

- Schließlich die Vereine. Sie sind in vielen Fällen kommerzialisiert oder überaltert. Sie sind zwar weiter wirksame kommunale Interessensinstrumente aber - unfreiwillig - selektieren sie in der Stadtgesellschaft mehr als es gut für sie ist. Wir freuen uns über türkische Familienväter in der freiwilligen Feuerwehr und über marokkanische Jugendliche im Fußballverein.

Wer das Verbindende, das Soziale, der europäischen Stadt wieder zum klingen bringen will, wer das Durcheinander der Wertvermittlung (nicht dessen Verlust!) wieder ordnen will, braucht einen Ordnungsrahmen, der in die heutige Dualität von Staat und Wirtschaft eingreifen kann. Die Wohlfahrtsgesellschaft geht dann schon über den Wohlfahrtsstaat hinaus, weil sie die Erbringung (!), nicht die Verteilung der Sozialleistungen zum Gegenstand aller in der Demokratie macht (Walzer).

\section{Der zivilgesellschaftliche Ansatz}

Dies ist die Zivilgesellschaft und das mit ihr verbundene bürgerschaftliche Engagement. Aber auch hier gilt: Den Beschwörungen müssten viel konkretere Schritte folgen und die Veränderungen des »Gesamtorchesters « müsste analysiert werden. Zivilgesellschaft ersetzt keine Politik und ist kein Ornament (Münkler), sie ist "mehr Möbel als die Wohnung selbst, aber gerade deshalb lebensbestimmend «. (5) In der Stadtgesellschaft stößt dies auf mehr Konflikte als die Staatsund Reformtheoretiker vermutet haben.

Institutionen sind langlebig. Die Sicherheiten von Markt, Staat, Stadt, Nachbarschaft und Verein aufzugeben zugunsten neuer Aufgabenzuweisungen, neuer Spielregeln und neuer Statuszuweisungen in der Zivilgesellschaft macht Angst, verunsichert, kränkt und entlarvt Herrschaftsstrukturen (wie vor 30 Jahren - diesmal umgekehrt in der Absicht hektische Über- komplexität zu reduzieren, um wieder wirkliche Teilhabe aller in den Blick zu nehmen).

Viele kritische Beobachter von Kafka über Lapassade (6) bis Walzer nennen das die Aufgabe der Neuvermessung eines »virtuellen « Landes. Unsichtbare Grenzen aufheben - »Entgrenzung « nennen das die Soziologen (7), um neue Grenzziehungen vorzunehmen, bewusster, politischer und vergleichsweise rationaler. Dieses Bild zieht sich bei gesellschaftlichen Umbrüchen in der Geschichte durch.

In Städten kann das sehr konkret werden. Kreative Stadtentwicklung kann bedeuten, die "Sozialräume « neu oder erstmals zu vermessen, um gesellschaftlichen Teilhabeideen zum Lichte zu verhelfen, Bürgerengagement auf einer relevanten Akteursebene einzufügen - nicht als Ehrenamt »anzuhängen «. Auch die Diskussionen zum Quartiersmanagement haben das gezeigt.

Zur Neuvermessung der sozialen Stadtlandkarte gehört das Verhältnis zwischen Stadt und Vereinen. Sie brauchen neue "Zwischenlandschaften «, Projekte der Erneuerung, Öffnung mit neuen Rollen. Im Verein wird man Mitglied, in Bürgerschaftsprojekten wird man Pate, Botschafter, Mentor.

Die Neuvermessung zwischen Staat und Stadt wird bei allen neueren politischen Programmen der letzten Jahre in Deutschland zur unlösbaren Zusammenfügung der Gegensätze: Mit der Reform der Sozialgesetzbücher II und XII entstehen neue Behörden wie die ARGE für Beschäftigung mit unterschiedlichen Logiken in der gleichen Institution. Sie ist ebenfalls ein neuer Akteur im städtischen Orchester.

Mit der neuen Familienpolitik des Bundes (Krippen, Mehrgenerationenhäuser) wird Landespolitik »überholt «. Städte haben beim Vermessen der Kindergartenlandschaft deshalb verschiedene Maße zu benutzen. Einmal nach vermeintlich tatsächlichem Bedarf (Nachfragepolitik des Landes) und einmal nach erwünschenswertem Sollbedarf (Bund). Ungesicherte Institutionen wie die Mehrgenerationenhäuser sind Marken eines neuen Institutionenverständnisses, bei dem sich Nutzen aus der jeweils konkreten Energie und Ressource im Stadtteil für alt und jung speist und nicht aus festgesetzten Platzzahlen. 


\section{Generationenkonflikte?}

Der Generationenvertrag der deutschen Sozialpolitik, das unsichtbare aber wirkungsvolle füreinander zahlen über Sozialabgaben, ist selbst ein Mischprodukt von Staat und Zivilgesellschaft. Unter dem Druck der Ökonomisierung stellt ihn die jüngere Generation immer mehr in Frage, weil es letztlich auch eine Vertrauensfrage ist, ob diese Generation sich wiederum auf die nachfolgende Generation verlassen kann. Gleichzeitig laufen im privaten Bereich gegenseitige Finanztransfers von jung und alt in großem Umfang.

Der Leistungsdruck setzt nun schon 50Jährige frei auf dem Arbeitsmarkt und verstärkt die Illusion, dass »die Alten « entweder viel kosten, weil sie sich mit guten Renten privatisieren oder viel kosten, weil sie arbeitslos und später pflegebedürftig sind. Immer häufiger fehlt auch die "selbstverständliche" Betreuungsgroßmutter für den Enkel, sodass intergenerative Beziehungen prekärer geworden sind. Weder allein privat noch allein staatlich kann eine lebendige Generationenbeziehung hergestellt werden. Zwar haben sich parteipolitisch Generationsabspaltungen (»Graue Panther « oder Jugendparteien) nicht durchgesetzt, aber der gelingende Alltag bei Betreuung, Nachbarschaft und im Vereinswesen ist komplizierter geworden.

Statt des »Generationenkrieges « gib es eine Funkstille, auch weil die Lebensentwürfe der Menschen unterschiedlicher Alter nebeneinander, aber nicht miteinander laufen. Es häufen sich Irritationen bei jüngeren Erwachsenen, welch verblüffende Alltagsentscheidungen ältere Menschen " plötzlich « treffen.

Auf der kommunalen Ebene ist die Vorstellung, der Stadtjugendring und der Seniorenbeirat sollten kontinuierlich zusammenarbeiten, noch kaum vorstellbar. In Kindergärten ist die Idee eher die Ausnahme, ältere Menschen, die nicht verwandt sind mit den betreuten Kindern, sollten neben Kindern, Fachpersonal und Eltern eine Rolle spielen und wirft misstrauische Fragen auf, welche Motive vorherrschen.

Es benötigt deshalb mehr als bisher Handlungsräume, in denen sowohl ältere Menschen eine produktive Rolle im Generationenverhältnis spielen als auch zwischen den Generationen selbstverständlicher nachbarschaftlich Hand angelegt wird. Die Mehrgenerationenhäuser ermöglichen, darüber zu sprechen und Alltagsbeispiele auszutauschen.

\section{Institutionen einer kreativen Stadt}

Neue Institutionen können so neue Wert- und Familienmuster aufbauen, stehen aber unvermittelt zu den alten Institutionen und ihren Regeln. Sie werden starkes Bürgerengagement brauchen, damit sie die »Leuchttürme « neuer »Landstriche" auf der städtischen Sozialkarte auch zum Leuchten bringen können. Dies wird erhebliche kreative Anstrengungen erfordern.

Bürgerengagement auf ungesichertem Terrain ist meist autonomes Engagement; an den Staat adressiert, punktuell, komplementär. Eine erwartbare bürgerschaftliche Dienstleistung, dem Vereins- oder Patenengagement vergleichbar, braucht eine Ordnungsstruktur, ein geregeltes, produktives Zusammenspiel. Dies ist bei einem solchen neuen Konzept auch noch bereichsübergreifend $\mathrm{zu}$ überdenken (Kita/Senioren; marktwirtschaftlich/gemeinnützig etc.).

Aus der reinen Idee für eine bessere familienpolitische Welt allein erwächst bestenfalls eine kleine Ideenbotschaftergruppe oder eine Lobby-Vereinigung von Verbandsvertretern. Wenn solche neuen Institutionen wie das Mehrgenerationenhaus dauerhaft ihre Rolle im städtischen Orchester spielen sollen, brauchen sie verbindliche Vernetzungen, offensichtliche Nutzerstrategien und die Unterstützung im politischen Mikroklima einer Stadt. Solche Institutionen müssen kooperieren in einem Gefüge, in dem Vereine miteinander Arbeitsteilung machen, Nachbarschaften offen sind, Städte Treffpunkte eröffnen, der Sozialstaat Schnittstellen dazu hat und die Wirtschaft den Nutzen erkennt. Wenn dies nicht der Fall ist, wird diese Idee eine zusätzliche und tendenziell belanglose Institution.

Wenn in anderen Feldern jedoch Projekte vernetzbar sind, wirkt ein intergenerativer Treffpunkt potenzierend und bestärkend. In den Städten wird es Bündnisse geben müssen, die solche Wechselwirkungen planen, kommunizieren und ermöglichen.

In Augsburg (270.000 Einwohner) haben wir mit dem »Bündnis für Augsburg « ein Dach geschaffen, in dem neue Projekte angeschoben, aber auch zugeordnet werden. So ist an der Schnittstelle von Kindergarten und Integrationspolitik ein Modell der Stadtteilmütter entstanden (über 400 Frauen), die in ihrer Muttersprache Kinder unterrichten und darauf bauen, dass mit dem gleichen Text in deutsch die klassische Institution Kindergarten die Zweisprachigkeit sichert. Mit Mentoren sichern wir den Freiwilligeneinsatz der Schüler (»Change-in «) zwischen Schule und Institution (Einsatzstelle). Ähnlich bei Sozialpaten und Demenzpaten, Sorgentelefonmütter und der Teamgruppe Neubürgerempfang. Alle arbeiten auf Augenhöhe mit der klassischen Institution (gleich ob Amt für Soziale Leistungen, Krankenhaus, Referat Oberbürgermeister usw.) und erweitern diese um ihre Funktion und Identität.

\section{Moderne Menschenbilder}

Wir fügen Funktionen und Kompetenzen neu zueinander - Teil des neuen Klangs der kreativen Stadt. Wir fügen im engen Sinne nicht Menschen als solche neu zueinander - das würde die Ängste im städtischen Raum aktivieren, dass mit jedem Projekt freiwillig engagierte Menschen dort verloren gehen, wo sie zuvor aktiv waren. In den primären Netzwerken unserer Städte ist viel Zurückhaltung gegenüber bürgerschaftlichen Projekten. Sie werden in einem Nullsummenspiel wahrgenommen, quasi zu Lasten vorhandener Verbindlichkeiten. Genau deshalb greift die Zivilgesellschaft auf moderne Strukturen multipler Identitäten zurück.

Menschen können gleichzeitig in ihren Vereinen sein und sich zu Paten ausbilden. Walzer dreht das Bild als Chance der Zivilgesellschaft um und sagt, dass niemand nur als Vertreter einer Gruppe oder Ethnie handelt, sondern als Person verschiedene Loyalitäten unter einen Hut bekommen kann. (8) Die Stadtteil- und Sorgentelefonmütter können sich im Bündnis für Augsburg engagieren, ohne dass in ihrer (Migrations- ) Familie jemand aus dem Moscheeverein austreten muss. Und eine Seniorenbeirätin kann sagen, dass sie genauso die Interessen der Kinder im Mehrgenerationenhaus vertritt, wie sie weiter für Senioren tätig ist. Ein katholischer Kirchengemeinderat kann in einem evangelischen Selbsthilfeprojekt helfen. 
Wichtig über solchen Projekten ist jedoch ein Dach, eine Meta-Identität, beispielsweise in Augsburg eine parteiübergreifende Steuerungsgruppe zugunsten des Gesamtmottos »Wir sind Augsburg «. Ohne solche Einrahmungen würden Projekte zu interaktiven Hilfs- und Beziehungsmaßnahmen (etwa des Mittelstandopas für die alleinerziehende Mutter) »verkommen «. In einem Zivilprojekt der kreativen Stadt spielt die alleinerziehende Mutter als Bürgerin im Orchester in ihrer Art an der gleichen Weise mit wie der ältere Mann an anderer Stelle. Wir brauchen Institutionen, in den viel mehr Menschen als bisher an der Erbringung von Wohlfahrt praktisch-kreativ mitwirken können. (9)

Die Kooperationsbereitschaft auf den hier dargestellten Institutionsebenen kommt dort zustande, wo die Analyse gemacht wurde, dass jeder für sich die gesellschaftlichen Ziele allein nicht mehr erreicht. Macht allein stiftet keinen Sinn mehr; der Staat allein sorgt nicht mehr Solidarität; die Stadt allein garantiert nicht mehr die vielkulturelle Identität; der Stadtteil allein sichert keine Toleranz; Vereine allein erzeugen nicht mehr Gemeinschaftsgefühle. Wer immer noch glaubt, mit Geld, Macht oder Moral sich über diese Analyse wegsetzen zu können, gibt die falschen Signale an die Bürgerschaft, deren Vertrauen wir brauchen.

\section{Stadt der Generationennetzwerke}

Aus solchen Grenzen erwachsen Notwendigkeiten zur Kooperation. Wo dies beispielsweise Bundes- oder Landesbehörden nicht begriffen haben, bleibt Kooperation taktisch und ohne Zugewinn von Bürgerengagement auf lokaler Ebene.

Die kreative Stadt steuert dies nicht zentralistisch und schiebt nicht alles selbst an, sondern sucht sich jeweils Partner zur Umsetzung. Allerdings können dies auch neue Partner sein - von der städtischen Tochterfirma über ein Wirtschaftsunternehmen, eine Bürgerinitiative bis zu einem Obstbauverein, der Jugendarbeit macht.

Kommunalpolitik muss die Initiierung neuer sozial-kreativer Politik zu einer Führungsaufgabe auf Dezernentenebene machen. Die Städte bauen damit die neuen Wohlfahrtsmixstrukturen auf, die in einer älter werdenden Gesellschaft mehr Ressourcen erschließen und Sinn stiften.
(10) Der Mix ist nicht hauptsächlich dafür da, mehr Ressourcen hinzuzuziehen, sondern die soziale Institution sinnhafter zu machen.

Den Zusammenhalt der Stadt, der Identität der europäischen Stadt, zu sichern, bedarf etwa der gleichen Pflege wie wir es kennen von Umwelt- oder Denkmalschutzpolitik, ist unverzichtbar eine aktuelle, moderne bürgerschaftliche Managementaufgabe. Am Beispiel Augsburger Kommunalpolitik fügen wir Steuerungselemente der städtischen Sozialpolitik, des bundespolitisch gestützten Mehrgenerationsmodellen und des Bürgerschaftsengagements im "Bündnis für Augsburg "Schritt für Schritt zusammen aber nach einem »Masterplan « kreativer Stadtentwicklung.

Auch ein Biotop braucht systematische Planung von Freiräumen und Eigenverantwortung. Mitscherlich nannte das schon früh »die Stadt als Geburtsort der Freiheit « (11), aus der heraus Menschen wiederum in Verbundenheit kreativ sich engagieren.

\section{Anmerkungen}

(1) Jürgen Habermas: Die neue Unübersichtlichkeit, Frankfurt am Main 1985.

(2) »Wie wir sehen werden, gehört es zu jedem erfolgreichen System der Toleranz, dass es nicht von einer bestimmten Form dieser Tugend abhängt, es verlangt nicht, dass all seine Teilnehmer sich an einem Punkt des Spektrums befinden. « Michael Walzer, Über Toleranz, Berlin 1998.

(3) »Der Begriff der Entwicklung hat mithin eine nicht nur doppelte, sondern widersprüchliche Bedeutung erlangt: nämlich herrschaftlich-urbane Machbarkeit gegenüber Natur und Menschen als Sinn und Ziel geistiger wie materieller Tätigkeit anzustreben, ohne die Kosten an Lebensglück oder Lebensqualität der Abhängigen wirklich einzubeziehen. « Ernest Jouhy, Klärungsprozesse, Frankfurt am Main 1988.

(4) Konrad Hummel: Öffnet die Altersheime, Weinheim 1982. Ders.: Wege aus der Zitadelle, Hannover 1986. Ders.: Freiheit statt Fürsorge, Hannover 1991. Ders.: Bürgerengagement, Freiburg im Breisgau 1995.

(5) »Die Zivilgesellschaft ist kein bloßes Ornament der politisch-gesellschaftlichen Ordnung, mit dem man sich schmücken, auf das man zur Not aber auch verzichten kann, sondern es handelt sich um eine notwendige Komplementarität, ohne die eine jede politisch-gesellschaftliche Ordnung aus der Balance kommt. «Herfried Münkler, Was bewegt die Zivilgesellschaft?, Bonn 2006.

(6) »Der Landvermesser Kafkas ist nicht wirklich Landvermesser. Er misst überhaupt nichts. Man glaubt zwar, dass er deshalb da sei, in Wirklichkeit aber stört er und stiftet Unruhe, ein Fremder, der die Ordnung des verschlafenen Dorfes durcheinander bringt. « Georges Lapassade, Der Landvermesser, Stuttgart 1976.

(7) Ulrich Beck, Christoph Lau (Hg.), Entgrenzung und Entscheidung: Was ist neu an der Theorie reflexiver Modernisierung? Frankfurt am Main 2004.

(8) Die funktionale Ausdifferenzierung der modernen Gesellschaft trennt schließlich nicht Personen voneinander; sie unterscheidet und unterteilt vielmehr verschiedene Hinsichten der einen sozialen Wirklichkeit: diverse Rationalitätsdimensionen von sozialer Interaktion bzw. deren Steuerung, die wir (mehr oder weniger) alle beherrschen (müssen). »Anders als die ständische, erwartet die moderne Gesellschaft von den Individuen, sich auch gegenüber all diesen relativ autonomen Wertsphären, denen jeder Individuum in seinen >Rollen als Citoyen, Bourgeois, Kirchenmitglied u. ä. jedoch zugleich unterworfen bleibt, als >Person< zu begreifen, zu definieren, zu verhalten. « Michael Walzer, Über Toleranz, Berlin 1998.

(9) »Der Wohlfahrtsstaat koexistiert mit der Wohlfahrtsgesellschaft, aber die Gesellschaft ist heute schwach im Vergleich zum Staat. Eine lebendige und helfende Wohlfahrtsgesellschaft, die von einem starken Wohlfahrtsstaat unterstützt, aber nicht kontrolliert ist, das würde eine fundamentale Änderung in den Beziehungen der Verteilung darstellen. « Michael Walzer in den Blättern der Wohlfahrtspflege 12/1995.

(10) Leopold Rosenmayr, Schöpferisch Altern. Eine Philosophie des Lebens, Wien 2007.

(11) »Es ist aller Mühen wert, weil die Menschheit, wie sie geworden ist, in den Städten ihre Wurzeln hat. Die Stadt ist der Geburtsort dessen, was wir bürgerliche Freiheit nennen, dieses Lebensgefühls, das sich dumpfen Herrschaftsgewalten widersetzte." Alexander Mitscherlich, Die Unwirtschaftlichkeit unserer Städte, Frankfurt am Main 1967. 